\title{
A UNIFIED TREATMENT OF SOME BASIC PROBLEMS IN HOMOTOPY THEORY ${ }^{1}$
}

\author{
BY PETER I. BOOTH \\ Communicated by E. H. Spanier, September 5, 1972
}

0 . Introduction. This note announces some new methods in algebraic topology, based on the results of $[\mathbf{1}],[2]$ and [3]. The relative lifting problem [5, p. 415] is fundamental to that subject. It includes the extension problem, the retraction problem, the lifting problem, the section problem, the relative section problem and the computation of $[X, Y]$ problem, among its particular cases. These problems (excluding the last one) are usually taken to concern just the existence of extensions, retractions, etc.; we use the terms in the wider sense, to include both the existence question and the homotopy classification question, for the respective extensions, retractions, etc. We will:

(i) prove that many cases of the above problems (including the computation of the absolute and relative cohomology groups of the total space of a fibration, and the computation of certain homotopy groups) are equivalent to "parallel problems", involving the restricted fibered mapping projection $(p q ; a)$;

(ii) give some examples of solutions of parallel problems. Our solutions include one lifting problem and two extension problems. The extension results have some immediate consequences; they give new derivations for the exact cohomology sequences of Serre and Wang. Gysin's sequence is derived separately. Future papers will discuss these and other applications in detail.

Our argument is valid in several convenient categories, including the category of $\mathfrak{f}$-spaces [4], [2], [7] and the category of quasi-topological spaces [6], [1], [2]. This category of $\mathfrak{f}$-spaces, for a definition see $[\mathbf{2}$, p. 276], contains the usual category of Hausdorff $k$-spaces ( = compactly generated spaces $=$ Kelley spaces) as a subcategory.

1. Preliminaries. $\approx$ will be used to denote bijections and isomorphisms; $\cong$ to denote homeomorphisms. We outline the concepts from [1], [2] and [3] that are required. If $X$ and $Y$ are spaces, then $\mathscr{M}(X, Y)$ will denote the space of maps of $X$ into $Y$; if $f \in \mathscr{M}(X, Y)$ then $\mathscr{M}(X, Y ; f)$ will denote the path component of $f$ with the subspace topology.

AMS (MOS) subject classifications (1970). Primary 55F05, 55F20, 55G40, 54C35, 54D50; Secondary 55B20, 55E05.

Key words and phrases. Fibration, section, lifting, extension, cohomology, homotopy, $k$-spaces, exponential law of maps.

${ }_{1}$ Partially supported by National Research Council of Canada Grant A8023. 
Let $B$ be a simply connected space. If $p: X \rightarrow B$ and $q: Y \rightarrow B$ are maps then $f: p \rightarrow q$ will mean that $f: X \rightarrow Y$ is a map such that $q f=p . \mathscr{M}(p, q)$ will denote the set of maps $f: p \rightarrow q$, topologized as a subspace of $\mathscr{M}(X, Y)$. Let $X_{b}$ and $Y_{b}$ denote the fibres of $p$ and $q$ over a point $b \in B . p \sqcap q: X \sqcap Y \rightarrow$ $B$ and $(p q):(X Y) \rightarrow B$ will denote the associated pullback map and the associated fibred mapping projection, respectively (their fibres over $b$ are $X_{b} \times Y_{b}$ and $\mathscr{M}\left(X_{b}, Y_{b}\right)$, respectively). We recall the exponential law of maps: $\mathscr{M}(p \sqcap q, r) \cong \mathscr{M}(p,(q r))$. If $q$ and $r$ are fibrations, i.e. have the absolute covering homotopy property in the sense of the category used, then so is $(q r)$. (qr) is a rather inconvenient fibration to work with, for its fibres are not in general path-connected. Let $A$ denote the set of homotopy classes of maps $Y_{b} \rightarrow Z_{b}$, for a given choice of $b \in B$. $(Y Z ; a)$ will denote the path component of $(Y Z)$ that contains $a \in A$. Then $(p q ; a)=(p q)$ $(Y Z ; a)$ is a fibration over $B$ with path-connected fibres. The condition that $\pi_{1}(B)=0$ is not strictly necessary, the essential point is that $B$ can be given a base point such that $\pi_{1}(B)$ acts trivially on the path components of the distinguished fibre of $(q r)$.

2. Main theorems. Let $f, g \in \mathscr{M}(p, q)$. We say that $f$ is homotopic to $g$ over $B$ if there is a homotopy $H: f \simeq g$ such that $H_{t} \in \mathscr{M}(p, q)$, for each $t \in I$. $[p, q]$ will denote the set of such homotopy classes.

THEOREM 1. If $X$ is path-connected then there is a bijection $[p \sqcap q, r] \approx$ $\bigcup_{a \in A}[p,(q r ; a)],[f] \mapsto[g]$, defined by $f(x, y)=g(x)(y) ; p(x)=q(y)$.

PROOF. $[p \sqcap q, r] \approx[p,(q r)] \approx \bigcup_{a \in A}[p,(q r ; a)]$.

This means that the lifting problem for $p \sqcap q$ over $r$ is equivalent to a set of lifting problems, for $p$ over maps $(q r ; a)$.

Let $B_{0}$ be a nonempty subspace of $B$. We define $X_{0}=p^{-1}\left(B_{0}\right)$, $Y_{0}=q^{-1}\left(B_{0}\right), Z_{0}=r^{-1}\left(B_{0}\right), p_{0}=p\left|X_{0}, q_{0}=q\right| Y_{0}$ and $r_{0}=r \mid Z_{0}$. Let $h \in \mathscr{M}\left(p_{0}, q_{0}\right) . \mathscr{M}(p, q)_{h}$ will denote the space of all maps $f \in \mathscr{M}(p, q)$ such that $f \mid X_{0}=h$. We will say that $f$ is h-homotopic to $g$ over $B$ if there is a homotopy $H: f \simeq g$, such that $H_{t} \in \mathscr{M}(p, q)_{h}$, for each $t \in I .[p, q]_{h}$ will denote the set of such homotopy classes. Let $u: p_{0} \sqcap q_{0} \rightarrow r_{0}$. If $X_{0}$ is path-connected then the corresponding map $v: p_{0} \rightarrow\left(q_{0} r_{0}\right)$ takes $X_{0}$ into a single path component $(Y Z ; a)$ of $(Y Z)$, thus giving a map $w: p_{0} \rightarrow\left(q_{0} r_{0} ; a\right)$.

THEOREM 2. If $X$ is path-connected then there is a bijection

$$
[p \sqcap q, r]_{u} \approx[p,(q r ; a)]_{w}, \quad[f] \mapsto[g],
$$

defined by $f(x, y)=g(x)(y) ; p(x)=q(y)$.

PROOF. $[p \sqcap q, r]_{u} \approx[p,(q r)]_{v} \approx[p,(q r ; a)]_{w}$. 
This asserts that the relative lifting problems, for the diagrams (i) and (ii), are equivalent.

(i)

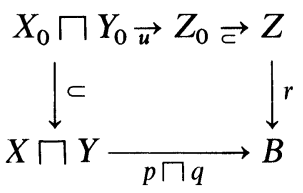

(ii)

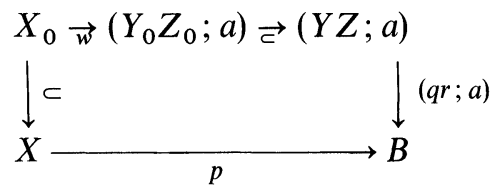

\section{Basic problems.}

(I) The lifting problem as a set of section problems. Let $p=1_{B}$, and $q$ and $r$ be fibrations. It follows from Theorem 1 that $[q, r] \approx\left[1_{B} \sqcap q, r\right] \approx$ $\bigcup_{a \in A}\left[1_{B},(q r ; a)\right]$. Hence the lifting problem for one fibration over another is equivalent to a set of section problems, for fibrations $(q r ; a)$.

(II) The computation of $[Y, K]$. If $Y$ and $K$ are spaces then $[Y, K]$ will denote the set of homotopy classes of maps $Y \rightarrow K$. This can be taken either in the "free" sense or in the base-point preserving sense. Our method is relevant to the case where $Y$ is the total space of a fibration $q: Y \rightarrow B$. Let $t: K \times B \rightarrow B$ denote the usual projection. If $f: Y \rightarrow K$ is a given map, then there is an associated map $g: Y \rightarrow K \times B, g(y)=(f(y)$, $q(y)), y \in Y$; now this carries over to homotopy classes giving $[Y, K] \approx[q, t]$. It follows from $(\mathrm{I})$ that $[Y, K] \approx \bigcup_{a \in A}\left[1_{B},(q t ; a)\right]$.

Hence the computation of $[Y, K]$ is equivalent to a set of section problems. In particular, (a) if $K$ is an Eilenberg-Mac Lane space $K(\pi, n)$, the computation of $H^{n}(Y ; \pi)$ corresponds to the solution of a set of section problems; (b) if $q$ is a Hopf fibration $\left(Y=S^{n}\right)$ then the computation of $\pi_{n}(K)$ corresponds to the solution of a set of section problems.

(III) The relative lifting problem as a relative section problem. Let $q: Y \rightarrow B$ and $r: Z \rightarrow B$ be given fibrations and $B_{0}$ be a nonempty pathconnected subspace of $B$. If $g: q_{0} \rightarrow r_{0}$ then $(Y Z ; g)$ will denote the path component of $(Y Z)$ that contains the restrictions of $g$ to the fibres over $B_{0}$. If 1 denotes the identity on $B_{0}$ then we take the composite $1 \sqcap q_{0} \cong$ $q_{0} \vec{g} r_{0}$ and define $h: 1 \rightarrow\left(q_{0} r_{0} ; g\right)$ as the associated map. We will replace $q$ by $1_{B} \sqcap q$ and apply Theorem 2 . It follows that the relative lifting problem for diagram (iii) is equivalent to a relative section problem for the fibration ( $q r ; g)$ (i.e. the relative lifting problem for diagram (iv)).

(iii)

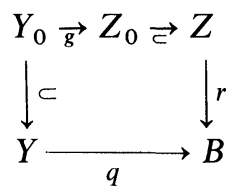

(iv)

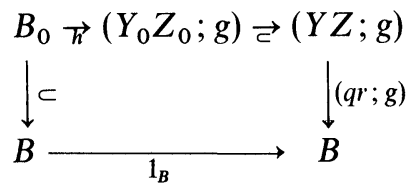

(IV) The extension problem. Let $K$ be a given space. If $f: Y_{0} \rightarrow K$ is a given map then there is an associated map $g: Y_{0} \rightarrow K \times B_{0}, g(y)=$ $(f(y), q(y)), y \in Y_{0}$. Consider the relative lifting problem of diagram (iii) 
above, with $r$ replaced by the usual projection $t: K \times B \rightarrow B$. The extension problem for fover $Y_{0} \subset Y$ is equivalent to that relative lifting problem and hence to the corresponding relative section problem for $(q t ; g)$. The retraction problem for $Y_{0} \subset Y$ can, of course, be treated in this way.

Notation. Let $X$ and $B$ be spaces with base points and $p: X \rightarrow B$ be a base-point preserving map. [sec $p$ ] will denote the set of based homotopy classes of base-point preserving sections to $p$.

(V) The relative cohomology of the total space of a fibration. We will now take $B_{0}$ to be a single point, the base point of $B ; F$ will denote the distinguished fibre for the fibration $q$. Let $\pi$ be an abelian group. We take $K$ to be the Eilenberg-Mac Lane space $K(\pi, n)$ and $f=0: F \rightarrow K(\pi, n)$ to be the constant map value zero. This map will be taken to be a base-point in $(Y K(\pi, n) \times B ; 0)$, its path-component in $(Y K(\pi, n) \times B)$. The elements of $H^{n}(Y, F ; \pi)$ are in one-to-one correspondence with the homotopy classes of extensions of 0 over $Y$; it follows via (IV) that they are in oneto-one correspondence with the elements of $[\sec (q t ; 0)]$.

4. Techniques for the solution of parallel problems. Some parallel problems are extremely difficult, others are relatively easy. We give a few solutions. They are not on the level of maximal interesting generality; our main concern is to emphasize the existence of the methods used. Example (ii) is a particular case of (I) above; examples (i), (iii) and (iv) are cases of $(\mathrm{V})$.

(i) Serre's exact cohomology sequence. Consider the map $f^{\prime}: t \sqcap q \rightarrow t$, $f^{\prime}(u, y)=u ; t(u)=q(y)$. It follows, via the exponential law, that there is a corresponding map $f: t \rightarrow(q t ; 0)$; the restriction of $f$ to the distinguished fibres is

$$
g: K(\pi, n) \rightarrow \mathscr{M}(F, K(\pi, n) ; 0), \quad g(a)(x)=a, \quad a \in K(\pi, n), x \in F .
$$

It is easily shown that if $F$ is $(m-1)$-connected, then $g$ induces isomorphisms of homotopy groups in all dimensions $\geqq n-m+1$.

Proposition. Let $B$ be a pointed CW-complex and $p_{i}: X_{i} \rightarrow B$ be fibrations with distinguished fibres $F_{i}(i=1$ and 2$)$. The $p_{i}$ will be assumed to be base-point preserving. $f: p_{1} \rightarrow p_{2}$ is a base-point preserving map, such that $g=f \mid F_{1}: F_{1} \rightarrow F_{2}$ induces isomorphisms of homotopy groups in all dimensions $\geqq j$. If the connectivity of $B$ is $\geqq j$ then $f$ induces a bijection $f_{*}:\left[\sec p_{1}\right] \rightarrow\left[\sec p_{2}\right]$.

This proposition is a modification of $[5$, p. 418, Theorem 12].

Putting $p_{1}=t, p_{2}=(q t ; 0)$ and assuming that the connectivity of $B$ is $\geqq n-m$, we see that $f$ induces a bijection $f_{*}:[\sec t] \rightarrow[\sec (p t ; 0)]$. Now since $[\sec t] \approx H^{n}(B ; \pi),[\sec (p t ; 0)] \approx H^{n}(Y, F ; \pi)$, it follows that $p^{*}$ : $H^{n}(B ; \pi) \rightarrow H^{n}(Y, F: \pi)$ is an isomorphism. We use [5, p. 412, Theorem 1] 
to eliminate the condition that $B$ is a CW-complex; Serre's exact cohomology sequence is then easily derived.

This technique can be extended to cover other problems, the essential point is to find a fibration $p_{1}$ which "approximates" the given $p_{2}=(p q ; a)$, in the sense of our proposition or the relative version of that proposition. We now give another such example.

(ii) A lifting problem. Let $G$ and $\pi$ be abelian groups, $m$ and $n$ be positive integers, $n \leqq 2 m-1$. Any map $g: K(G, m) \rightarrow K(\pi, n)$ can be desuspended, i.e. there exists a map $h: K(G, m+1) \rightarrow K(\pi, n+1)$ such that $\Omega h \simeq g$. Let $q$ denote the principal fibration, fibre $K(G, m)$, induced by $c: B \rightarrow$ $K(G, m+1)$. Let $r$ denote the principal fibration, fibre $K(\pi, n)$, induced by $d: B \rightarrow K(\pi, n+1)$.

We define $p_{1}$ to be the principal fibration, fibre $K(\pi, n)$, induced by the map $d-h c: B \rightarrow K(\pi, n+1)$. ( $Y Z ; g)$ will denote the path-component of $g$ in $(Y Z)$; we take $g$ as the base-point for the space $(Y Z ; g)$. If the connectivity of $B$ is $>n-m$, then $p_{1}$ approximates to $p_{2}=(q r ; g)$, in the sense of the above proposition. The map $p_{1} \rightarrow p_{2}$ is defined by attaching one path to the end of another. The following conditions are then equivalent:

(a) There is a map $q \rightarrow r$, whose restriction to the distinguished fibres is $g$;

(b) There is a base-point preserving section to $(q r ; g)$;

(c) There is a section to $p_{1}$;

(d) $d \simeq h c$.

It follows that there is a lifting of $q$ over $r$ if and only if there is a cohomology operation $\theta \in \Theta(\pi, m+1 ; G, n+1)$ such that $\theta([c])=[d]$.

(iii) Wang's exact cohomology sequence. Let us assume that the fibration $q: Y \rightarrow B$ has $B=S^{m}$. It follows from (V) above that the constant map $Y \rightarrow K(\pi, n)$ value 0 , corresponds to a distinguished section to $(q t ; 0)$. Hence the exact homotopy sequence for $(q t ; 0)$ splits and $\pi_{m}(Y K(\pi, n) \times B ; 0)$ is canonically isomorphic to $\pi_{m}(B) \oplus \pi_{m}(\mathscr{M}(F, K(\pi, n) ; 0))$. A section to $(q t ; 0)$ is a map $S^{m} \rightarrow(Y K(\pi, n) \times B ; 0)$; it determines an element of $\pi_{m}(Y K(\pi, n) \times B ; 0)$ and the canonical projection gives a corresponding element of

$$
\pi_{m}(\mathscr{M}(F, K(\pi, n) ; 0)) \approx \pi_{0}\left(\mathscr{M}\left(F, \Omega^{m} K(\pi, n)\right)\right) \approx H^{n-m}(F, \pi) .
$$

It follows from (V) that $H^{n}(Y, F ; \pi) \approx H^{n-m}(F, \pi)$; Wang's exact sequence is easily derived.

This technique may be extended, in a modified form, to cover other problems about fibrations over $H$-cogroups [5, p. 39]. It may be used in problems concerning homotopy groups, as indicated in (II) (b) above.

(iv) Gysin's exact cohomology sequence. We assume that the fibration $q: Y \rightarrow B$ has distinguished fibre $S^{m}$. The fibration $(q t ; 0)$ has distin- 
guished fibre $\mathscr{M}\left(S^{m}, K(\pi, n) ; 0\right)$, this space has the weak homotopy type of $K(\pi, n) \times K(\pi, n-m)$. Let $t_{1}: K(\pi, n-m) \times B \rightarrow B, t_{2}: K(\pi, n+1) \times$ $B \rightarrow B$ denote the usual projections. The first stage in a Moore-Postnikov factorization of $(q t ; 0)$ involves a map $g:(q t ; 0) \rightarrow t_{1}$; the $k$-invariant of the second stage is a map $h^{\prime}: K(\pi, n-m) \times B \rightarrow K(\pi, n+1)$. We define $h: t_{1} \rightarrow t_{2}$ by $h(x, b)=\left(h^{\prime}(x, b), b\right), x \in K(\pi, n-m), b \in B$. Let $f: t \rightarrow(q t ; 0)$ denote the map defined in (i) above. There is a sequence of fibrations $\cdots \rightarrow t \rightarrow_{f}(q t ; 0) \rightarrow_{\mathrm{g}} t_{1} \rightarrow_{h} t_{2} \rightarrow_{f}\left(q t_{2} ; 0\right) \rightarrow \cdots$ inducing $\cdots \rightarrow[\sec t] \rightarrow_{f_{*}}$ $[\sec (q t ; 0)] \rightarrow_{g_{*}}\left[\sec t_{1}\right] \rightarrow_{h_{*}}\left[\sec t_{2}\right] \rightarrow_{f_{*}}\left[\sec \left(q t_{2} ; 0\right)\right] \rightarrow \cdots$. Now $[\sec t] \approx$ $H^{n}(B, \pi),[\sec (q t, 0)] \approx H^{n}(Y, F ; \pi)(\operatorname{see}(\mathrm{V})$ above $),\left[\sec t_{1}\right] \approx H^{n-m}(B, \pi)$ and $\left[\sec t_{2}\right] \approx H^{n+1}(B, \pi)$. It follows that there is an exact sequence:

$$
\cdots \rightarrow H^{n}(B, \pi) \rightarrow H^{n}(Y, F ; \pi) \rightarrow H^{n-m}(B, \pi) \rightarrow H^{n+1}(B, \pi) \rightarrow \cdots .
$$

5. Remark. "Ordinary" cohomology can be defined in terms of homotopy classes of maps into Eilenberg-Mac Lane spaces; a number of mathematicians have suggested that this is the best definition. The above results are consistent with this approach. More generally, our arguments fit nicely into a discussion of generalized cohomology, defined using a spectrum.

\section{REFERENCES}

1. P. I. Booth, The exponential law of maps. I, Proc. London Math. Soc. (3) 20 (1970), 179-192. MR 40 \#6490.

2. - The section problem and the lifting problem, Math. Z. 121 (1971), 273-287.

3. - The exponential law of maps. II, Math. Z. 121 (1971), 311-319.

4. A. Clark, Quasi-topology and compactly generated spaces, Mimeographed Notes, Brown University, Providence, R.I. (unpublished).

5. E. H. Spanier, Algebraic topology, McGraw-Hill, New York, 1966. MR 35 \# 1007.

6., Quasi-topologies, Duke Math. J. 30 (1963), 1-14. MR 26 \# 1847.

7. R. M. Vogt, Convenient categories of topological spaces for algebraic topology, Proc. Adv. Study Inst. Alg. Top., 42.1-42.7, Aarhus, Denmark, 1970.

DePartment of Mathematics, Memorial University of Newfoundland, St. John's, NEWFOUNDLAND, CANADA 